\title{
Time series Doppler imaging using STELLA
}

\author{
Michael Weber, Klaus G. Strassmeier, and Thomas Granzer \\ Astrophysikalisches Institut Potsdam, An der Sternwarte 16, D-14482 Potsdam, Germany \\ email: mweber@aip.de
}

\begin{abstract}
One of the core programs of the STELLA robotic observatory is to monitor the stellar activity on a sample of stars using Doppler imaging. We present first preliminary results of the rapidly rotating, single giant star HD 31993 from the first two years of operation. We confirm the presence and orientation of differential rotation on the stellar surface.
\end{abstract}

Keywords. Instrumentation: spectrographs - techniques: spectroscopic - stars: imaging - stars: rotation - stars: spots

\section{Observations}

All observations were carried out at the STELLA robotic observatory using the STELLA echelle spectrograph (SES) which is fed with an optical fiber from one of the two STELLA $1.2 \mathrm{~m}$ telescopes (Weber et al. 2008). We accumulated approximately 170 spectra of HD 31993 during the last 2 years. Since it is a winter target, it was first observed in winter 2007, again during the winter 2007/2008 and is now being observed for the third round. The average $\mathrm{S} / \mathrm{N}$ ratio is 250 , but has been significantly lower during a period of bad fiber alignment (spring to summer 2007). We derived heliocentric radial velocities by cross-correlating 20 orders of each spectrum with a template spectrum of similar spectral type. Since the line-profiles are considerably deformed due to the surface activity of the star, it is difficult to reliably derive radial velocities without manual intervention. Therefore we spun-up the template spectrum before doing the cross-correlations, which resulted in very reliable measurements in unattended mode.

\section{Doppler imaging and differential rotation}

The data consists of three seasons so far. The first season (beginning of 2007) covers a bit more than 2 months, the second season half a year, and the third season has just begun. We concentrate on the second half of the second season, since the beginning of that season had many bad nights and also suffered from lower efficiency due to instrument alignment issues.

The data set used for the following analysis consists of 57 spectra taken between the third of December 2007 and the 19th of March 2008. This is a bit more than three stellar rotations at a rotation period of 25.3 days. The phase coverage is generally very good, depending only on the weather on the site, which was exceptionally good this last winter.

As in Strassmeier et al. (2003) we used TempMap (Rice \& Strassmeier 2000) to calculate the Doppler images. The primary goal was to compare the results with the previous ones, which were based on two spectral lines only. SES delivers the full optical spectrum, but in the red half of the spectrum inter-order gaps exist. Due to this, we did this comparison only with one spectral line (Ca I 6439) for now, since the other line (Fe I 6430) is right on the edge of two orders.

In Strassmeier et al. (2003), which was based on data from late 1996, the surface was dominated by spots at latitudes from $0^{\circ}$ to $40^{\circ}$. There was no feature at or near the pole 


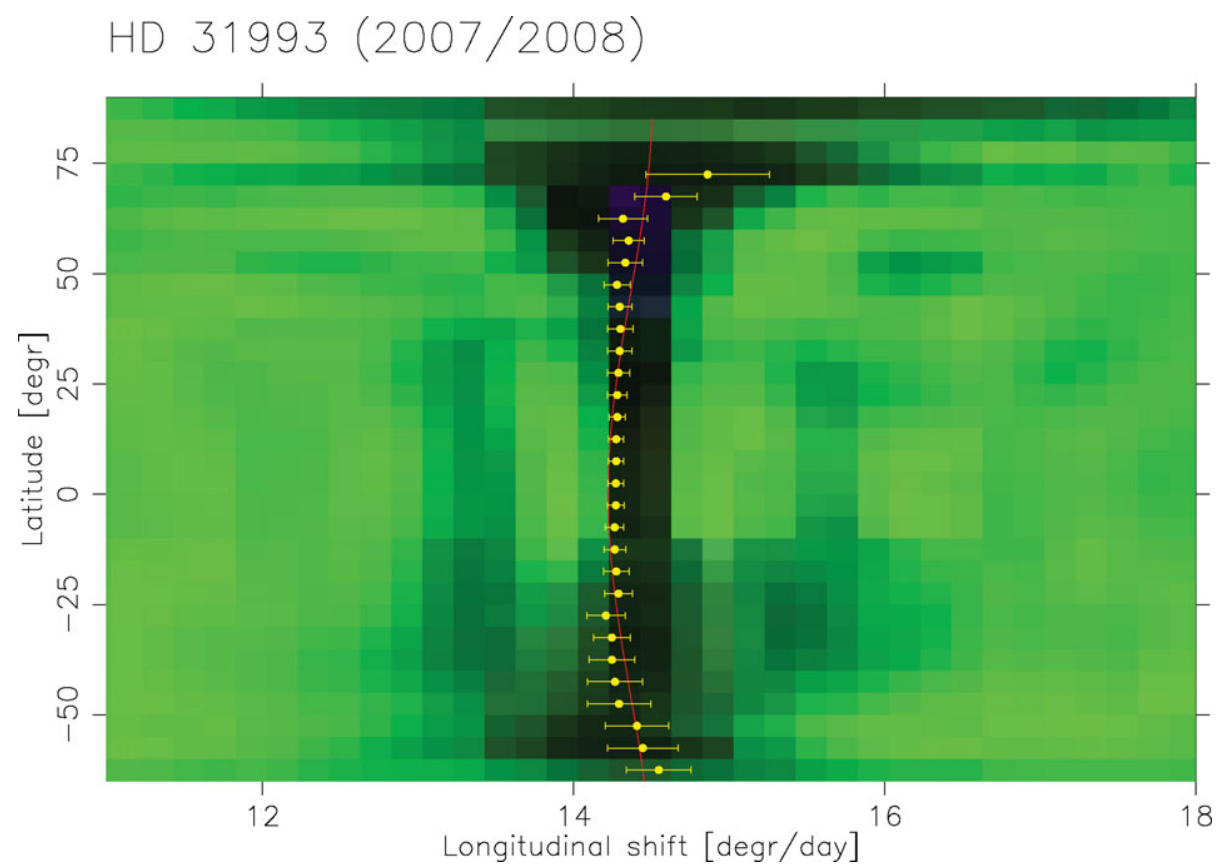

Figure 1. Average cross correlation of HD 31993. The red line is the fit $\Omega(b)=14.22+0.28 \sin ^{2}(b)^{\circ} /$ day.

then. Our new Doppler image is dominated by spots at lower latitudes, but also shows dark features at the pole and a bright ring at $65^{\circ}$. Since the latter corresponds exactly to the sub-observer latitude, it is likely that these features are caused by non-optimal values for the projected rotational velocity and/or the instrumental profile.

To derive a possible differential rotation, we cross-correlated all consecutive Doppler images of our data set. Using just two individual Doppler images does not give conclusive results, but is dominated by the noise in the spectra and/or short-term variations of spots on the stellar surface. We thus divided the data set, which consists of 57 spectra, into 16 individual chunks, and calculated a Doppler image for each of those. We then crosscorrelated the individual consecutive images with each other (e. g. the first with the sixth, the second with the seventh, etc.), which gave eleven cross-correlation images. The average of these eleven images, each adjusted for small variations in the time difference between the data chunks, is shown in Fig. 1.

The image reveals almost rigid rotation, the relative differential rotation is only $2 \%$, lap time is $360 . / 0.28=1285$ days. This is much weaker than the $12 \%$ found by Strassmeier et al. (2003), but the values derived here are based on only one spectral line and are thus preliminary. Using many of the lines available in the SES spectra will give a more precise measurement of this star's differential rotation.

\section{References}

Rice, J. B. \& Strassmeier, K. G. 2000, $A \& A S$ 147, 151

Strassmeier, K. G., Kratzwald, L., \& Weber, M. 2003, A\& A 408, 1103

Weber, M., Granzer, T., Strassmeier, K. G., \& Woche, M. 2008, in Advanced Software and Control for Astronomy II, ed. A. Bridger \& N. M. Radziwill, SPIE 7019, 70190L 